\title{
Trabajo infantil y deserción escolar en el Perú al 2015
}

\author{
Child labor and school desertion in Perú by 2015
}

\author{
Michael Tovar Obregón ${ }^{1 *}$, Claudia Rios Cataño ${ }^{1}$ \\ ${ }^{1}$ Universidad Continental, Huancayo
}

\section{RESUMEN}

La investigación tuvo como objetivo analizar el trabajo infantil y la deserción escolar con base en informaciones del Instituto Nacional de Estadísticas e Informática Perú 2015. Se trató de un estudio retrospectivo descriptivo-correlacional, con datos obtenidos de la página del Instituto Nacional de Estadísticas e Informática (INEI), la muestra estuvo conformada por niños entre 5 a 9 años de edad, haciendo un total 717 encuestados, los datos descriptivos fueron analizados en el programa estadístico SPSS versión 24, y para las asociaciones se utilizó el programa EViews aplicando la prueba de Máxima Verosimilitud del modelo Probit Bivariado con un $p \leq 0,05$. Los resultados mostraron que los niños que realizaron actividades laborales fueron un $54,4 \%$ (257) de sexo masculino y el $47,6 \%$ (233) de sexo femenino, así la mayoría 84,5 \% (414) pertenecían al área rural y el 15,5\% (76) área urbana, la mayoría residían 58,2 \% (285) residían en la región Sierra, en cuanto a la deserción escolar del total de niños el $95 \%$ (681) refirió que estudia con regularidad a la escuela, mientras que el $5 \%$ (36) dejó de asistir, se encontró una relación directa entre el trabajo infantil y la deserción escolar, con un coeficiente de correlación $(0,2315)$, entre la asistencia al colegio y el hecho de trabajar con una probabilidad de ocurrencia del 20,42\%. Se concluye que el trabajo infantil es predominante en área rural y residentes de la región sierra, así también existe asociación estadística entre el trabajo infantil y la deserción escolar.

Palabras clave: Trabajo infantil; deserción escolar; actividad laboral.

\section{ABSTRACT}

The research has the objective to analyze child labor and school dropout base don information from the National Institute of Statistics and Informatics Perú 2015. It was a retrospective descriptive correlational study, wth data obtained from the National Institute of Statistics and Informatics (INEI), the sample consisted of children between 5 to 9 years of age, making a total of 717 surveyeds, the descriptive data were analyzed in the statistical program SPSS version 24, and for associations the EViews program was used and the Maxium Likelihood test of the Maxium Likelihood test of the Bivariate Probit model with a $p \leq 0,05$ was used. The results showed that the children who carried out work activities were 54,4\% (257) of male sex and $47,6 \%$ (233) of female sex, the majority $84.5 \%$ (414) belonged to the rural área and $15,5 \%$ (76) urban área, most reside 58,2\% (285) resided in the Sierra región, in ítems of school dropout of the total number of children $95 \%$ (681) reported that they regularly study the school, while $5 \%$ (36) stopped attending, a direct relationship was found between child labor and school dropout, with a correlation coefficient $(0,2315)$, between attending school and working with a probability of ocurrence of $20,42 \%$. It is concluded that child labor is predominant in rural áreas and residents of the mountain región, so there is also a statistical association between child labor and school dropout.

Keywords: Child labor; school dropout; work activity.

Historial del artículo:

Recibido, 10 de mayo de 2017; aceptado, 4 de junio de 2017; disponible en línea, 25 de junio de 2017

* Bachiller de Economía.

Correo: michaeltovarobregon@gmail.com 


\section{INTRODUCCIÓN}

Millones de niños trabajan en todo el mundo, en muchos casos esto es un obstáculo para su educación, desarrollo y porvenir, se estima que el $20 \%$ de la población mundial de niños entre 5 y 17 años son económicamente activos, así también esta situación de trabajo puede poner al niño en riesgo físico y psicológico, incluso puede poner en riesgo sus vidas, esto se puede considerar como una violación de los derechos del niño que perpetua la pobreza y compromete el crecimiento económico y el desarrollo equitativo, aunque también existe un debate si el trabajo infantil debe ser erradicado por completo o si se puede considerar con un derecho del niño, pues se considera que es un problema económico porque reduce la acumulación de capital humano y puede afectar su productividad e ingresos futuros (GRADE, 2017), (OIT, 2017).

Según la Organización Internacional del Trabajo (OIT, 2017), en América Latina la participación laboral de niños y niñas de 7 a 12 años representa el $27 \%$ del total del trabajo de menores de edad. En Perú, el trabajo involucra al $16 \%$ de los niños y adolescentes que se hallan entre los 6 y los 17 años de edad, los pequeños entre 6 y 13 años que laboran, el $67,5 \%$ se ubican en zonas rurales, asimismo, las regiones que presentan mayor concentración de trabajo infantil son Huancavelica, Puno, Huánuco, Amazonas y Pasco y en Junín.

El trabajo infantil es un fenómeno complejo y multidimensional con componentes sociales, culturales y económicos, así también está determinado por situaciones, actitudes e incluso valores que pueden predisponer a las familias a aceptar e incluso a aceptar el trabajo infantil en algunos contextos especialmente en la clase pobre, para algunos padres toman este tipo de actividades como una escuela de vida, como una forma de capacitar a sus hijos en términos de habilidad, conocimientos y para enseñarles lo que es la vida, por otra lado también existen explicaciones de por qué los niños deben de trabajar en lugar de estudiar que van desde factores estructurales y culturales sobre todo vinculadas con la situación socioeconómica de hogares en pobreza, pasando por la permanencia de costumbres y normas culturales que toman al trabajo infantil como una inversión (Sandoval-Avila, 2007).

Respecto a la deserción escolar se ha demostrado que existe relación entre el trabajo infantil y la exclusión educativa, los menores de edad que trabajan presentan un retraso de dos a tres grados con relación a los menores que no trabajan, también existe diferencia entre la deserción escolar en la educación primaria y media siendo superior la deserción en el nivel secundario, con predominio del sexo femenino (Sandoval-Avila, 2007).
La Encuesta de Trabajo Infantil (ETI), se realiza cada 7 años siendo la última en 2014 y publicada de manera oficial en 2015, esta ha sido especialmente diseñada para el estudio del trabajo infantil y adolescente, captura adecuadamente el porcentaje de menores que se encuentran inmersos en actividades laborales, algunas de sus particularidades con respecto a otras encuestas es que incluye informaciones directas e indirectas, así también este estudio es a nivel nacional, Nacional Urbano, Nacional Rural, Costa, Sierra, Selva y Lima Metropolitana (INEI, 2017).

El presente estudio tuvo algunas limitaciones a pesar de que los datos fueron extraídos de la ETI, entre ellas está el tamaño de muestra que no fue homogénea para poder realizar estadísticas por departamentos, así también la encuesta tiene una gran cantidad de espacios en blanco y falta de preguntas excluyentes, mismo así es una fuente de información oficial realizada a nivel nacional.

Considerando lo antes mencionado se tuvo como hipótesis que la deserción escolar está relacionada con el trabajo infantil siendo que esta relación está afectada de manera directa por el género, área y región.

Con base en lo expuesto el presente estudio tuvo como objetivo analizar el trabajo infantil y la deserción escolar con base en informaciones del Instituto Nacional de Estadísticas e Informática (INEI), Perú 2015.

\section{MATERIAL Y MÉTODOS}

Se trata de un estudio retrospectivo descriptivocorrelacional, con datos obtenidos de la página institucional del Instituto Nacional de Estadísticas e Informática (INEI) a través de la Encuesta Nacional Especializada en Trabajo Infantil (ETI).

La ETI se realizó del 29 de octubre al 21 de diciembre del 2015 en 24 departamentos incluyendo al Callao como Provincia Constitucional, dentro del ámbito urbano y rural del país. La población estuvo conformada por los niños y adolescentes entre 5 a 17 años de edad, haciendo un total 6239 encuestados.

Para el estudio se tomó en cuenta el cuestionario aplicado a niños de 5 a 9 años, se utilizó como criterio de exclusión las informaciones incompletas y preguntas respondidas por los padres, por lo tanto, la muestra se conformó por 717 niños.

Los datos descriptivos fueron analizados en el programa estadístico Statistic Package for the Social Science (SPSS) versión 24, para las variables cuantitativas se trabajaron medidas de variabilidad y medidas de tendencia central, para las variables 
cualitativas frecuencias y porcentajes, para realizar las asociaciones se utilizó programa EViews el método de Máxima Verosimilitud del modelo Probit Bivariado con un coeficiente de $p \leq 0.05, \%$, los valores negativos son interpretados como relaciones directas.

\section{RESULTADOS}

Se encontró que del total niños encuestados (717) entre 5 a 9 años, la edad media fue de 7,66 años, mediana de 8 y una desviación de 1,27, de los cuales $49 \%$ (351) fueron de sexo femenino y el $51 \%$ (366) de sexo masculino, en cuanto al sector se observó que el $85 \%$ (607) estaban en el ámbito rural y el 15 $\%$ (110) urbano, en cuanto a la región, sierra $59,1 \%$ (424), selva $33,5 \%$ (240) y costa 7,4 \% (53), en cuanto al departamento la mayor cantidad de encuestados a
Tabla 1. Actividades laborales desempeñadas por los niños, INEI, ETI, 2015.

\begin{tabular}{lrr}
\hline & $\mathrm{n}$ & $\%$ \\
\hline Agricultura & 421 & 85,9 \\
Mercado & 36 & 7,3 \\
Ambulante & 12 & 2,4 \\
Restaurant & 11 & 2,2 \\
Construcción & 10 & 2,0 \\
Domestico & 5 & 1,0 \\
Otras actividades & 12 & 2,4 \\
\hline
\end{tabular}

En cuanto a los motivos de trabajo, los niños refirieron que el principal motivo era ayudar a sus padres con $86,1 \%$ (422) así también varios infantes mencionaron dos o más motivos por cuales trabajaron, como muestra la tabla 2. Entre tanto los niños refirieron que sólo un $2,8 \%$ (20) trabajaban para ellos mismos el

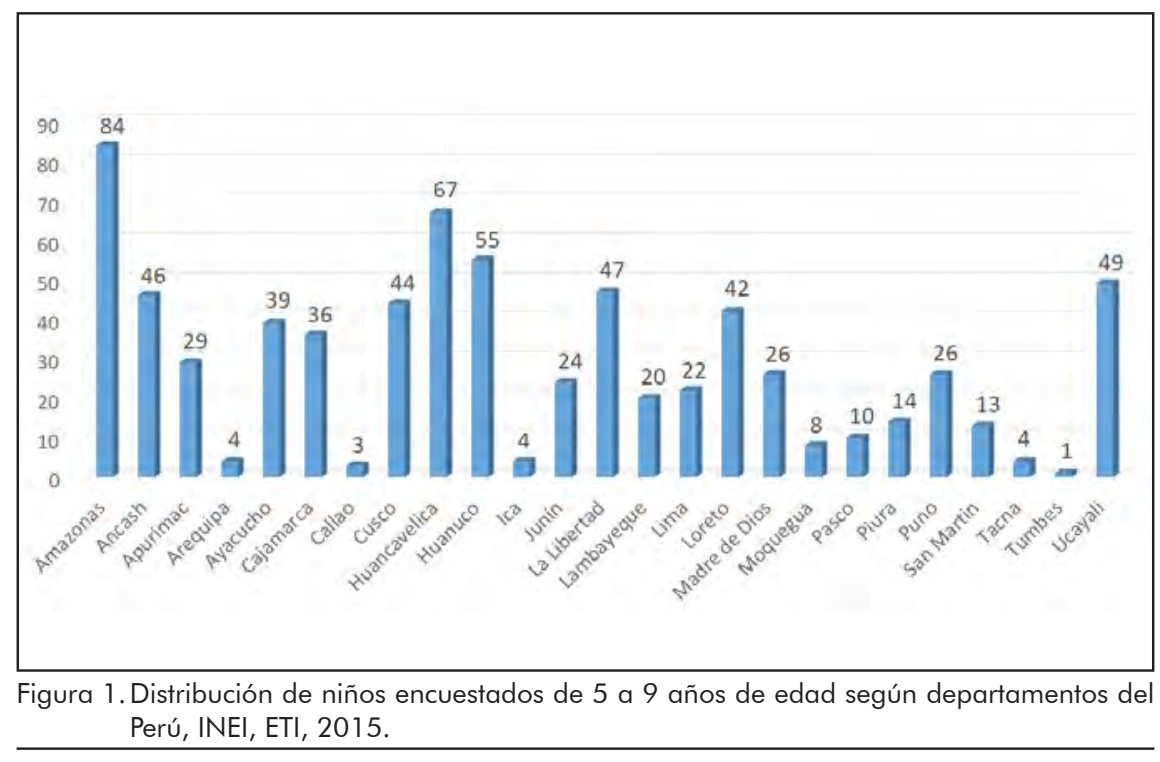

nivel nacional se encontraron en los departamentos de Amazonas, Huancavelica, Huánuco y Ucayali, como se puede observar en la figura 1.

En cuanto a la variable de trabajo infantil el $68 \%$ (490) de niños trabajan, en tanto que el $32 \%$ (227) no trabajan; del total de niños que realizaron actividades laborales el 54,4\% (257) fueron de sexo masculino y el $47,6 \%$ (233) de sexo femenino, así también se encontró que la mayoría 84,5\% (414) pertenecían al área rural y el 15,5\% (76) área urbana, así también la región el 58,2 \% (285) residían en la región Sierra, el 35,9 \% (176) de la región Selva y el 5,9 \% (29) región Costa, en cuento al trabajo infantil cabe destacar que los niños en varios casos desempeñaron más de una actividad laboral siendo que la mayoría trabajaba en el sector de la agricultura con un $85,9 \%(421)$ y en menor proporción en otras actividades (tabla 1). resto es lo hacen para otras personas.

Tabla 2. Motivos del trabajo infantil en niños, INEI, ETI, 2015.

\begin{tabular}{lrr}
\hline & $\mathrm{n}$ & $\%$ \\
\hline Ayuda a los padres & 422 & 86,1 \\
Gusta trabajar & 161 & 32,9 \\
Aprender el oficio & 71 & 14,5 \\
Para comprar algunas necesidades & 39 & 3,5 \\
Ganar dinero & 33 & 6,7 \\
Otro motivo & 17 & 3,5 \\
\hline
\end{tabular}

En relación a la deserción escolar del total de niños el $95 \%$ (681) refirió que estudia con regularidad a la escuela, mientras que el $5 \%$ (36) dejó de asistir, del total de alumnos que abandonaron los estudios fueron 
$50 \%$ para cada género, por otro lado, el 83,3 \% (30) fueron del área rural y el 16,7 \% (6) del área urbana, en cuanto a la deserción por regiones se registró un $47,2 \%$ (17) de la sierra, $41,7 \%$ (15) de la selva y de la costa un $11,1 \%$ (4). No se respondieron las preguntas relacionadas a si repitieron de grado así como no

Tabla 3. Correlación entre trabajo infantil, deserción escolar, género y área de residencia en niños, INEI, ETI, 2015.

\begin{tabular}{lrrrr}
\hline Variable & Coeficiente & Std.Error & z-Statatic & Probabil. \\
\hline Deserción & $-1,775628$ & 0,250656 & $-7,083918$ & 0,0000 \\
Trabaja & 0,231565 & 0,182372 & 1,269736 & 0,2042 \\
Género & $-0,007351$ & 0,159075 & $-0,046213$ & 0,9631 \\
Área & $-0,047280$ & 0,214413 & $-0,220507$ & 0,8255 \\
\hline
\end{tabular}

había datos relacionados a su desempeño académico.

El presente trabajo mostró una relación directa entre el trabajo infantil y la deserción escolar (tabla 3), tuvo un coeficiente de correlación $(0,2315)$, entre la asistencia al colegio y el hecho de trabajar con una probabilidad de ocurrencia del 20,42 \%. También existe correlación negativa $(-0,0073)$ entre la deserción y el género, siendo que el género femenino se relaciona con deserción con una probabilidad de ocurrencia de $96,31 \%$. Así también se encontró relación negativa $(-0,0472)$ según área, donde el sector más vulnerable es el rural con una probabilidad de ocurrencia de $82,55 \%$.

\section{DISCUSIÓN}

El trabajo infantil no es un problema nuevo, desde hace mucho tiempo a los niños y adolescentes se les ha exigido su participación en una gran cantidad de trabajos, desde los más sencillos hasta los más extenuantes en donde incluso perdían la vida por las fatigosas tareas a las que eran sometidos (GRADE, 2011). En el presente estudio se aprecia el trabajo infantil en ambos sexos, con ligero predominio del sexo masculino, de manera marcada se aprecia mayor proporción de trabajo infantil en el sector rural y las regiones de la sierra y selva, estos resultados coinciden con el estudio de Sapelli (2004), el autor menciona que los padres de las zonas rurales asignan mayor valor a la asistencia a la escuela en los niños comparado con las niñas, se considera que los padres demandan menos educación para las hijas mujeres, además que la educación aumenta el nivel de gasto para el hogar.

Se considera que las tasas de actividad laboral infantil son cerca del $42 \%$ de los niños del grupo de edad 5-17 años que realizan actividades laborales, mientras que esta tasa se eleva a más del $50 \%$ para los adolescentes de 12 a 17 años (OIT, 2002). En cuanto a la asistencia a la escuela cerca del 13
$\%$ de adolescentes no asisten a un centro educativo, cuando por ley estarían obligados a estar cursando los estudios secundarios, según las respuestas de los niños, manifestaron que estudian y trabajan, de tal manera que la deserción escolar es mínima, mismo así en la encuesta de la ETI, no hace mención respecto al rendimiento académico de los niños que laboran y estudian, tampoco hubieron preguntas relacionadas con el tiempo que dedican al estudio o si han repetido el año de estudio.

Existe una creciente población de niños que trabajan desde muy temprana edad y que viven día a día en situación de alto riesgo, tanto físico como moral. Siendo las principales formas de explotación trabajos en terrenos agrícolas, trabajos callejeros, domésticos y sexuales, en el presente estudio queda en evidencia que la mayoría de los niños trabaja en la sector de la agricultura, con menor porcentaje en mercados y trabajos ambulantes, hay que considerar que los niños del presente estudio tenían una edad entre 5 y 9 años, lo cual limita otras formas de trabajo.

Según Salazar (1996) los niños que trabajan en general son de familias pobres, pero también existen factores culturales, al parecer todos los miembros de la familia tienen que ser proveedores de manera económica mediante el trabajo y la responsabilidad que se les forma para que sean adultos competentes del mañana, los padres justifican el trabajo de sus menores aduciendo que adquieren valores como la responsabilidad, autonomía y la tenacidad para sobrellevar las dificultades o para soportar sacrificios, además lo consideran importante para evitar vicios como la ociosidad y la delincuencia.

En la encuesta de la ETI no apreciaron datos relacionados si el trabajo es remunerado o impuesto por los propios padres a manera de evitar gastos con contrato de personal. Hay tener en cuenta que los niños de la muestra por su edad deben realizar grandes esfuerzos físicos para cumplir con su trabajo laboral considerando que la mayoría trabaja en el sector de la agricultura.

Según referencia de los niños encuestados se encontró una minoría (5\%) de deserción escolar por motivo del trabajo, este dato no se ha corroborado con las estadísticas del Ministerio de Educación para tener con exactitud este dato, se ha documentado que el trabajo infantil y la escolaridad son excluyentes y afecta a los sectores más pobres de la población, así también el repetir el grado, el rendimiento académico, el atraso escolar y niños que finalizan sus estudios con varios años después son características que de niños trabajadores, en algunos países las jornadas laborales en niños y adolescentes superan a los adultos Salazar (1996). 
Se tiene como conclusiones que, el trabajo infantil predomina en la zona rural, en especial en la región sierra y en el sexo masculino.

Asimismo la deserción total de los alumnos se da en una minoría, siendo que los alumnos refieren que estudian y trabajan, por otro lado, no se tienen datos respecto al rendimiento académico o repetición de cursos por parte de los niños que estudian y laboran ya que es importante no solo verificar si estudian, sino que su rendimiento académico sea óptimo para garantizar sus competencias y tener posibilidades de estudios superiores en un futuro.

Y, existe relación directa entre el trabajo infantil y la deserción escolar en niños de educación primaria aceptándose la hipótesis de investigación.

\section{REFERENCIAS BIBLIOGRÁFICAS}

Trabajo adolescente y deserción escolar en el Perú. GRADE Grupo de Análisis para el Desarrollo [en línea].2011 [fecha de acceso 8 de abril de 2017]. URL disponible en:

http://www.juntos.gob.pe/modulos/mod infojuntos_V1/docs/139.pdf
Un futuro sin trabajo infantil. OIT Organización Internacional del Trabajo [en línea].2002 [fecha de acceso 8 de abril de 2017].URL disponible en: http://www.ilo.org/ipecinfo/product/download. do? type $=$ document\&id $=5665$

Sandoval-Avila A. Trabajo infantil e inasistencia escolar. Revista Brasileira de Educacion. 2007;12(34): 6880.

Perú: niños y niñas adolescentes que trabajan, 19932008. INEI instituto nacional de estadísticas e informática [en línea].2009 [fecha de acceso 13 de abril de 2017]. URL disponible en: https://www. inei.gob.pe/media/menurecursivo/publicaciones_ digitales/est/lib0875/libro.pdf

Sapelli C, Torche A. Deserción escolar y trabajo juvenil: 2 dos caras de una misma decisión?. Cuad Econ [Internet]. 2017 [citado 16 de abril 2017]; 41(1):173-198. URL disponible en: http://www. scielo.cl/pdf/cecon/v41n123/art01.pdf

Salazar MC. El trabajo infantil en América Latina. Revista Colombiana de Educación. [en línea].1996 [fecha de acceso 20 marzo de 2017]. URL disponible en: http://revistas.pedagogica.edu.co/ index.php/RCE/article/view/5397/4424 